\title{
A novel method for infant multiple breath washout: first report in clinical
}

\section{practice}

\section{Authors:}

Anna Shawcross, $\mathrm{MD}^{1,2}$

Clare S Murray, MD ${ }^{1,2}$

Katy Pike, PhD 3,4

Alex Horsley, $\mathrm{PhD}^{1,5}$

\section{Affiliations:}

1. Division of Infection, Immunity \& Respiratory Medicine, Faculty of Biology, Medicine and Health, University of Manchester

2. Department of Paediatric Respiratory Medicine, Royal Manchester Children's Hospital, Manchester, UK

3. UCL Great Ormond Street Institute of Child Health, University College London, London, UK

4. Department of Paediatric Respiratory medicine, Great Ormond St Hospital for Children NHS Foundation Trust, London, UK

5. Manchester Adult Cystic Fibrosis Centre, University Hospital of South Manchester, Manchester, UK

Address for correspondence: Dr Alex Horsley.

Division of Infection, Immunity \& Respiratory Medicine, Faculty of Biology, Medicine and Health, University of Manchester, $2^{\text {nd }}$ floor ERC, University Hospital South Manchester, Southmoor Road, Wythenshawe, Manchester M23 9LT. alexander.horsley@manchester.ac.uk

Funding: This study was funded by a grant from the North West Lung Centre Charity. This work was also supported by the NIHR Manchester Biomedical Research Centre. This research was supported by the NIHR Great Ormond Street Hospital Biomedical Research Centre. AH was supported by an NIHR clinician scientist award (NIHRCS012-13). This report therefore represents independent research funded by the NIHR. The views expressed are those of the authors and not necessarily those of the UK National Health Service, the NIHR or the UK Department of Health.

The Innocor analyser and prototype infant washout circuit were provided on loan from Innovision ApS (Odense, Denmark). Innovision were not involved in the design, conduct, analysis or reporting of this study. 
Keywords: Infant pulmonary function, multiple breath washout, lung clearance index, cystic fibrosis

Running Head: Feasibility study of a novel method for infant multiple breath washout

\section{Abstract:}

Background: Lung clearance index (LCI), measured using multiple breath inert gas washout (MBW) is a potentially useful test in infants with respiratory disease, particularly cystic fibrosis (CF). Clinical use is limited however by the need for specialist staff and equipment. We have previously described a novel method for infant MBW suitable for use outside of specialist laboratories. This study describes its performance in vivo in infants with CF and healthy controls, including a limited comparison with the respiratory mass spectrometer.

Methods: Children aged $<2$ years with CF and controls underwent MBW testing on a single occasion. Practical applicability of the system was determined by the number of successful duplicate tests, and within-subject repeatability.

Results: 25 children ( 7 with CF, 18 healthy controls, all sedated with chloral hydrate) attempted MBW. 20 patients (7 with CF) successfully underwent duplicate testing ( $80 \%$ success rate). Mean within-subject coefficient of variation for FRC was $7.2 \%$ and for $\mathrm{LCl} 5.9 \%$. Comparison of $\mathrm{LCl}$ with the mass spectrometer was limited . but gave very similar values for $\mathrm{LCl}$ and FRC in those patients who underwent technically adequate tests with both methods.

Conclusions: We have described a new MBW method that is feasible and reproducible in sedated infants. Results fall within the expected range, and well within accuracy limits set by international guidelines. This could provide a more accessible alternative to previously described systems for infant MBW, and overcomes many of the technical challenges inherent in conventional MBW. 


\section{Introduction:}

Lung clearance index ( $\mathrm{LCl}$ ), derived from multiple breath inert gas washout (MBW) is increasingly recognized as a useful marker of early lung disease (1-3). MBW is particularly appealing in infants, being performed during tidal breathing without complex respiratory manoeuvres(4). It has a potential role in a number of respiratory conditions, but has particular applicability in cystic fibrosis (CF); in recent years, both the widespread adoption of newborn screening and the development of disease-modifying treatments have driven the search for improved methods of detecting and monitoring early CF lung disease $(5,6)$. However, the clinical use of infant pulmonary function testing, including MBW, is limited by the lack of suitable equipment which can be used outside a specialised research laboratory(7).

We have previously described a novel method for performing MBW in vitro that is suitable for use in infants (8). The "Manchester method" for infant MBW avoids several of the major technical challenges in infant MBW, simplifying the process to enable it to be performed outside a specialist laboratory. Conventional MBW, whether measured using nitrogen washout or with an exogenous tracer gas, relies on the simultaneous measurement of flow and gas concentration. These measurements are then aligned and combined, a process that is especially challenging at rapid respiratory rates such as those seen in infants. Small errors in signal alignment or delays in signal response time can lead to significant inaccuracy in the calculation of functional residual capacity (FRC) and $\operatorname{LCI}(9,10)$. The Manchester method eliminates this source of inaccuracy, as flow is not directly measured, and expired gas is collected instead for volume measurement at the end of the 
test. By removing the need for a pneumotachometer this also significantly reduces dead space in the system, another important consideration for ensuring accuracy in infant MBW(10, 11). Finally, this method measures $\mathrm{SF}_{6}$ directly rather than calculating it indirectly from molar mass measurements, using a photoacoustic gas analyser which has been shown to be accurate at low concentrations(12). Recently, concerns have been raised that $4 \% \mathrm{SF}_{6}$, which is used for infant $\mathrm{MBW}$ with a mass spectrometer or ultrasonic flowmeter, may alter infant breathing parameters thus rendering results inaccurate (13); the Manchester method uses $0.1 \% \mathrm{SF}_{6}$ with a similar viscosity to air, eliminating this concern.

This method was assessed for accuracy in a realistic lung model, and found to have superior accuracy in vitro than previously described systems such as the ultrasonic flowmeter (8). In this feasibility study, we now describe the performance of the Manchester method in vivo, both in healthy infants and in infants with CF. We also describe a direct comparison with the respiratory mass spectrometer, currently considered the gold standard for infant $\operatorname{MBW}(1,2)$.

The objectives of this study were:

1. To assess practical feasibility of using the Manchester method for infant MBW in patients, determined by number of successful duplicate measurements in patients with CF and healthy controls.

2. To determine the need for sedation when using the method

3. To assess repeatability of the obtained lung function parameters (FRC and LCI) within patients, defined as the coefficient of variation (CV) of within-visit repeats

4. To compare lung function parameters ( $\mathrm{FRC}$ and $\mathrm{LCl}$ ) between healthy 
controls and infants with $\mathrm{CF}$, and to compare the performance and acceptability of the test in these two groups. 


\section{Methods}

\section{Subjects}

This was an observational study, involving MBW assessment on a single occasion. Three groups of infants (aged <2 years) were recruited to undergo MBW testing using the Manchester method:

1. Children with CF under the care of Royal Manchester Children's Hospital, Manchester, UK (RMCH) were recruited to undergo MBW testing using the Manchester method on a single occasion.

2. Children with CF undergoing routine pulmonary function tests including MBW using a respiratory mass spectrometer at Great Ormond Street Hospital for Children, London, UK (GOSH). This allowed comparison of the Manchester method with the accepted gold standard for infant MBW.

3. Control infants ( $\geq 34$ weeks gestation; never previously ventilated; no history of respiratory disease, confirmed lower respiratory tract infection or significant respiratory symptoms; no history suggestive of aspiration) who were already undergoing a procedure requiring sedation (e.g. MRI scan) at Royal Manchester Children's Hospital.

At the time of recruitment, infants with CF in Manchester were offered the option to attempt MBW testing unsedated or sedated with chloral hydrate $(100 \mathrm{mg} / \mathrm{kg}$ according to local hospital protocol). All infants tested at GOSH were sedated according to local protocol with $80-100 \mathrm{mg} / \mathrm{kg}$ chloral hydrate. Control infants were sedated with $100 \mathrm{mg} / \mathrm{kg}$ chloral hydrate prior to undergoing their planned procedure. MBW testing was performed immediately after the MRI scan, while the infant 
was still sleeping. Heart rate and oxygen saturations were monitored continuously for all sedated infants.

Background clinical and demographic data, body weight and crown-heel length were recorded for all subjects. Subjects were assessed for fitness to proceed prior to MBW testing, and were not tested within 3 weeks of a recent respiratory illness or exacerbation.

Ethical approval for the study was obtained from Greater Manchester East Research Ethics Committee (REC reference 15/NW/0581) and written informed consent obtained from the parents of all participating infants.

\section{Multiple Breath Washout Testing}

All subjects were tested supine, during quiet sleep $(14,15)$.

The infant MBW apparatus for the Manchester method has been described in detail previously (8) and is illustrated in figure 1. Briefly, the circuit consists of two bags connected by a series of rapidly responding pneumatic valves. A two-way non-rebreathing valve divides these into two separate circuits, the inspiratory and expiratory arms. A Rendell Baker facemask (Intersurgical, Wokingham, UK) sealed with therapeutic putty (Mobilis Rolyon, Patterson Medical, Nottinghamshire, UK) was attached to the non-rebreathing valve, with the gas sample needle placed close to the facemask. The circuit was connected to an Innocor photoacoustic gas analyser (Innovision, Odense, Denmark) 
with custom research software to control the operation of the pneumatic valves. This allowed the operator to switch between wash-in and washout configurations.

During testing, the wash-in bag was filled with $0.1 \% \mathrm{SF}_{6}$ mixture, supplied from a portable gas cylinder integral to the gas analyser. At the start of wash-in, the valves were arranged so that the patient inspired directly from the wash-in bag and breathed out to room air. $\mathrm{SF}_{6}$ concentration was measured and displayed continuously by the Innocor gas analyser, allowing the operator to determine when wash-in was complete. Once equilibrium was reached, the operator rearranged the valves to the washout configuration (via a single switch); the patient was then breathing room air, whilst all expired gas was collected in the washout bag. Washout was terminated automatically once $\mathrm{SF}_{6}$ concentration dropped below $1 / 40$ of the concentration detected at the start of wash-in, and the patient reverted to both inspiring and expiring room air via open circuits. FRC and cumulative expired volume (CEV) were calculated from the volume and $\mathrm{SF}_{6}$ concentration of the gas contained within the washout bag, and used to determine $\mathrm{LCl}: \mathrm{LCl}=\mathrm{CEV} / \mathrm{FRC}$ with appropriate adjustments for gas sample flow and BTPS, as described previously (8).

MBW traces were assessed to ensure technical adequacy and absence of air leak. FRC and LCl for each participating individual were then calculated from the mean of at least two technically acceptable measurements.

Following the MBW test, parents were asked to complete a questionnaire to determine acceptability of the procedure. Although previous authors have described parental experiences of 
participating in similar research (16), no previously-validated questionnaire directly applicable to the requirements of this study was identified. A questionnaire was therefore devised by the research team to determine acceptability of the procedure. Parents were asked to identify what aspects (if any) of the testing procedure were stressful or upsetting, and whether they would be happy for their infant to undergo the MBW testing procedure again in future.

\section{Statistical analysis}

Data were analysed using Prism (GraphPad Software Inc., CA, USA). Parametric data were expressed as mean (SD) and non-parametric data expressed as median with interquartile range. Differences between groups were assessed using a Chi-squared, unpaired t-test or the Mann-Whitney test. Intra-test repeatability was determined by calculating the coefficient of variation (CV) of the three recordings for each subject. For the comparison of patients and controls, outcomes were compared using an unpaired t-test. Based on previously published LCl data in CF infants, a minimum of 13 children was required in each group to detect a $10 \%$ difference between healthy children and children with CF with $90 \%$ power at the $5 \%$ significance level(2). Significance level was set at $p=0.05$. 


\section{Results}

\section{Feasibility of LCI using the novel method}

A total of 28 control infants were recruited to the study, of whom 14 successfully underwent at least 2 technically adequate $\mathrm{LCl}$ measurements (Table 1). Of those who did not complete testing, 9 control infants awoke either before or during their first $\mathrm{LCl}$ attempt. Parental consent was withdrawn for one, and in two cases the researcher decided not to proceed for reasons unrelated to the study itself. In two cases, technical issues meant that though the infants tolerated the test, a result could not be generated. Infants who woke before the test could be completed were noted to be significantly younger (median age 37 vs 73 weeks, $p=0.01$ ) and smaller (median weight $8.5 \mathrm{~kg}$ vs $10 \mathrm{~kg}, p=0.03$ ) than those who successfully completed it.

A total of 10 patients with CF were recruited to the study. In five cases an initial attempt was made to complete the test without sedation: none of these attempts were successful. Two families declined to proceed with sedation and a third infant was deemed unsuitable to sedate. The test was therefore successfully completed in 7 infants with CF. Four of these infants (57\%) were DF508 homozygotes, and 6 (86\%) were pancreatic insufficient. There were no significant differences noted between those patients with CF who successfully underwent testing compared to those who did not. 
Overall, neither an unsedated infant nor one waking from sedation underwent a successful MBW measurement. A total of 25 patients (18 healthy controls, 7 infants with CF) were deemed to be adequately sedated and in quiet sleep, and therefore attempted MBW testing. Of these, 20 children $(80 \% ; 13 / 18$ healthy controls and $7 / 7$ infants with CF) successfully underwent two and 14 infants $(56 \% ; 8 / 18$ healthy controls and 6/7 infants with CF) successfully underwent 3 or more technically adequate MBW tests.

A total of 18 washout traces (from a total of over 70 washouts performed) from 14 patients were excluded for the following reasons:

- Technical issues with the washout or wash-in circuit meaning result could not be generated ( $n=5$ tests from a total of 3 patients). Two of these resulted in a complete failure to obtain results for this patient, 1 was quickly rectified.

- Inadequate wash-in (defined as wash-in terminated before equilibrium in $\mathrm{SF}_{6}$ concentration had been reached) affecting 13 tests from a total of 11 patients. 9 of these patients also had $\geq 2$ acceptable tests.

\section{MBW outcomes}

Overall, for all patients included in the study CV for FRC was $7.2 \%$ and $\mathrm{CV}$ for $\mathrm{LCl} 5.9 \%$. When restricted only to the 14 patients who completed three technically acceptable tests, CV for FRC was $6.4 \%$ and $\mathrm{CV}$ for $\mathrm{LCl}$ was $5.3 \%$.

FRC and LCl data for healthy controls and CF infants are presented in Table 2 and illustrated in Figure 2. Although both measurements were higher in infants with CF, this difference did not reach statistical 
significance for either FRC (mean in healthy controls $19.5 \mathrm{ml} / \mathrm{kg}$, and in infants with CF $21.8 \mathrm{ml} / \mathrm{kg} ; \mathrm{p}=0.3$ ) or $\mathrm{LCl}$ (mean in healthy controls 6.5 and in infants with CF 7.0; $p=0.13$ ).

\section{Comparison with respiratory mass spectrometer}

Four patients underwent $\geq 2 \mathrm{MBW}$ tests with both the respiratory mass spectrometer and the Manchester MBW method. Though FRC appeared to correlate well in all cases, two patients showed good agreement in $\mathrm{LCl}$ and two patients significant disagreement. Further interrogation of the MBW traces revealed that in these two patients, wash-in using the Manchester method was inadequate, possibly due to time constraints in completing both sets of measurements. This was clearly identifiable from the wash-in $\mathrm{SF}_{6}$ trace, and resulted in $\mathrm{LCl}$ that was $20 \%$ higher when using the respiratory mass spectrometer, despite much smaller differences in FRC (10\% and 6.7\%). These tests would have been disregarded for clinical/research use after assessment for quality control. In the 2 cases where there was adequate performance of both methods, LCI differed by $<5 \%$ between the two methods.

\section{Acceptability to parents}

A total of 14 parents completed the questionnaire; 8 healthy controls and 6 infants with CF.

Before the test, $5 / 14$ (35\%) parents reported being worried about their infant being sedated; 3 of these were parents whose children were already being sedated for a planned procedure, rather than specifically for the MBW study. No parents reported being worried about the MBW test itself, or any other aspect of the study.

Following the test, only one parent reported finding the experience of their child being sedated distressing. No other aspect of the test process was rated as distressing or upsetting by parents. $13 / 14$ parents (93\%) reported that they would have been happy for their child to undergo the test again. From the free text box 
provided, the major emerging themes were that parents' main source of anxiety was sedation, rather than MBW testing itself, and secondly that the staff involved in the study played an important role in parental reassurance and acceptability of the test process overall.

\section{Discussion}

This is the first clinical report of the Manchester method for measuring $\mathrm{LCl}$ in infants.

The method described here has a number of advantages over alternative systems for infant MBW. It is simple and portable, allowing measurement of both FRC and $\mathrm{LCl}$ at the bedside. The removal of direct flow measurement leads to a highly accurate system(8), with a low deadspace consistent with international guidelines(11). The direct measurement of $\mathrm{SF}_{6}$ also avoids another potential source of error, as other systems rely on derivation of tracer gas concentrations from molar mass measurement which may lead to inaccuracies(17). The use of $0.1 \% \mathrm{SF}_{6}$ also eliminates concerns about higher concentrations (eg. $4 \%$ ) of tracer gas affecting the infant respiratory pattern and thus altering results (13). Finally, the testing process itself is straightforward and minimal expertise is required by the staff performing it. This would allow its use in centres without an existing pulmonary function laboratory, and indeed it was successfully introduced to just such a centre in this feasibility study. The experience of watching their child undergo MBW using this method was acceptable to parents.

The method does have some limitations. Since it does not measure flow directly, it can only provide calculated values for $\mathrm{FRC}$ and $\mathrm{LCl}$, with none of the additional measures which can be reported from standard MBW (e.g. moment ratios, phase 3 slope analysis) - though at present these are rarely used in infants and never outside the research context (18). The nature of the method also means that the maximum weight of patients which can be assessed is limited to around $12 \mathrm{~kg}$, since larger infants with larger lungs require washin and wash-out volumes in excess of the bag volumes used. Because the equipment does not incorporate 
a filter, it must also be disassembled for thorough cleaning between patients, limiting the number who can be assessed in a given day. Finally, the nature of the calculations used to determine FRC and LCI mean that it is possible to generate believable but inaccurate results from tests which have been inadequately washed in, therefore strict offline quality control is required before results can be fully interpreted. This is an important consideration in introducing the test into new centres, and would need to be taken into consideration if using it as a clinical tool.

Early technical issues with the study equipment were quickly rectified. These included faults with the equipment itself, human error in inexperienced staff and software flaws (specifically, tests terminated before being adequately washed in, due to image resolution on the computer screen). Difficulties of this nature are not unexpected in the first tests of any novel method, and success rates improved over the course of the study. Success rates in the second centre, which included comparison with the respiratory mass spectrometer, were lower. The primary cause of this appeared to be the lack of experience with the Manchester method in the second centre, together with time restraints due to the number of lung function assessments already scheduled. Although we were able to confirm that introduction of the method to a second site was feasible, as with any new technique success rates were lower in the initial period of use. Had the study run for longer in the second centre we would have expected to see higher success rates there.

Overall, however, despite these early challenges we were able to demonstrate high success rates $(80 \%$ success rate for those infants enrolled in the study who were adequately sedated at the time of commencing MBW testing).

In this study, we were unable to successfully measure $\mathrm{LCl}$ on any unsedated infants. The requirement for sedation is one of the major barriers to the more widespread adoption of all infant MBW techniques. MBW has been described in unsedated neonates and very young infants in natural sleep by several authors $(3,13$, 
19). Gray and colleagues described impressive success rates of $90 \%$ in a population-based cohort (age range 5-11 weeks) in rural South Africa, with a success rate of $65 \%$ for the same cohort aged 1 year $(20,21)$ This was only feasible however in the context of a well-staffed research study, which required high levels of patience and co-operation from families (including long visits whilst waiting for infants to achieve natural sleep) and a use of resources which is unlikely to be tolerated in clinical practice.

Initially we had hoped that some infants would tolerate MBW testing unsedated, hence this option was incorporated into the study protocol. However, this proved impossible, largely due to the practicalities of expecting children to sleep "on demand" during the daytime in an unfamiliar location. This may in part have been exacerbated by the weight and bulk of the equipment. Gray and colleagues used the lighter ultrasonic flowmeter. Infant MBW using a mass spectrometer has previously only been described in large cohorts of sedated infants $(1,2,22)$, and it is widely accepted in guidelines and clinical practice that sedation is required for all current infant lung function testing $(15,23,24)$. Since we aim for this method for infant MBW testing to be suitable for use in inexperienced centres, for both clinical practice and research, we advocate its future use with low dose sedation. In our study we used choral hydrate and this provided adequate sedation in all infants to carry out the test and therefore we would recommend low dose chloral hydrate as the sedative of choice. Other sedatives could be used but we have no first-hand experience as to whether the appropriate level of sedation would be achieved using a different preparation. Some centres use low dose propofol for infant lung function testing but this requires the additional cost of anaesthetic support for the procedure.

The results for FRC and $\mathrm{LCl}$ in health and disease obtained in this pilot study were consistent with those reported by studies using a respiratory mass spectrometer $(1,2)$. The largest study of infant MBW to date, published by Hoo et al in 2012, reported a mean FRC very similar to that reported here, but a slightly higher mean LCl of 7.46 in control infants and 7.89 in infants with CF. These data are now several years old, which may account for some improvement over time in the $\mathrm{CF}$ population $\mathrm{LCl}$, but it is also well recognised that the 
"normal range" for LCl varies according to the method used to measure it (25), (26). In particular, the small deadspace of the equipment used here would tend to lead to a lower normal range LCl. Furthermore, it is recognised that FRC may be underestimated by all MBW techniques (compared to that measured using plethysmography) and this may also contribute to a lower calculated LCI (2).

\section{Conclusion}

We have described a feasibility study of a novel method for performing MBW in infants. This method has been shown to be accurate in vitro and produces results in vivo which fall well within the acceptable limits for reproducibility set by international guidelines. Results are comparable to those described in the literature obtained using a respiratory mass spectrometer, and direct comparison in 2 patients showed the methods to generate similar results. . This method has the potential to allow sedated infant MBW in centres without expertise in infant lung function testing, or access to a specialist infant lung function laboratory.

\section{Acknowledgements:}

The authors would like to thank all infants participating in this study and their parents. The authors also thank Dr Anirban Maitra, Janet Edgar, Janice Fauset-Jones and the RMCH cystic fibrosis team for their support with the study. We also thank staff of the RMCH radiology department, particularly Yvonne Carrington, Jacqui Henderson and Ken Hindle, for their assistance with tests carried out on control infants. We also acknowledge the assistance of the NIHR Children's clinical research facility at Royal Manchester Children'. Finally, we wish to thank the staff of the cystic fibrosis unit at GOSH, particularly Dr Paul Aurora, and Dr Jane Kirkby, Stephanie Rees, Joanne Miles and Jacqueline Sutherland for their assistance in facilitating testing performed at GOSH. 



\section{References}

1. Lum S, Gustafsson P, Ljungberg H, Hulskamp G, Bush A, Carr SB, et al. Early detection of cystic fibrosis lung disease: multiple-breath washout versus raised volume tests. Thorax.62(4):341-7.

2. Hoo AF, Thia LP, Nguyen TT, Bush A, Chudleigh J, Lum S, et al. Lung function is abnormal in 3month-old infants with cystic fibrosis diagnosed by newborn screening. Thorax.67(10):874-81.

3. Hulskamp G, Lum S, Stocks J, Wade A, Hoo AF, Costeloe K, et al. Association of prematurity, lung disease and body size with lung volume and ventilation inhomogeneity in unsedated neonates: a multicentre study. Thorax.64(3):240-5.

4. Stocks J, Lum S, Ratjen RWWFBBCRD. 11 - Pulmonary Function Tests in Infants and Preschool Children. Kendig \& Chernick's Disorders of the Respiratory Tract in Children (Eighth Edition). Philadelphia: W.B. Saunders; 2012. p. 169-210.

5. Stick S, Tiddens H, Aurora P, Gustafsson P, Ranganathan S, Robinson P, et al. Early intervention studies in infants and preschool children with cystic fibrosis: are we ready? European Respiratory Journal.42(2):527-38.

6. Stick SM, Sly PD. Exciting new clinical trials in cystic fibrosis: infants need not apply. American Journal of Respiratory \& Critical Care Medicine.183(12):1577-8.

7. Davies G, Aurora P. The use of multiple breath washout for assessing cystic fibrosis in infants. Expert Review of Respiratory Medicine. 2017;11(1):21-8.

8. Shawcross A, Murray CS, Goddard N, Gupta R, Watson S, Horsley A. Accurate lung volume measurements in vitro using a novel inert gas washout method suitable for infants. Pediatric Pulmonology. 2016;51:491-497:.

9. Horsley A, Macleod K, Gupta R, Goddard N, Bell N. Enhanced photoacoustic gas analyser response time and impact on accuracy at fast ventilation rates during multiple breath washout. PLOS ONE [Electronic Resource].9(6):e98487.

10. Robinson PD, Latzin P, Ramsey KA, Stanojevic S, Aurora P, Davis SD, et al. Preschool Multiple-Breath Washout Testing. An Official American Thoracic Society Technical Statement. American Journal of Respiratory and Critical Care Medicine. 2018;197(5):e1-e19.

11. Robinson PD, Latzin P, Verbanck S, Hall GL, Horsley A, Gappa M, et al. Consensus statement for inert gas washout measurement using multiple- and single- breath tests. European Respiratory Journal.41(3):507-22.

12. Horsley AR, Gustafsson PM, Macleod KA, Saunders C, Greening AP, Porteous DJ, et al. Lung clearance index is a sensitive, repeatable and practical measure of airways disease in adults with cystic fibrosis. Thorax.63(2):135-40.

13. Banton GL, Hall GL, Tan M, Skoric B, Ranganathan SC, Franklin PJ, et al. Multiple breath washout cannot be used for tidal breath parameter analysis in infants. Pediatr Pulmonol. 2016;51(5):531-40.

14. Prechtl HFR. The behavioural states of the newborn infant (a review). Brain Research. 1974;76(2):185-212.

15. Stocks J, Sly PD, Tepper RS, Morgan WJ. Infant Respiratory Function Testing. New York, NY: John Wiley \& sons, Inc; 1996

16. Chudleigh J, Hoo AF, Ahmed D, Prasad A, Sheehan D, Francis J, et al. Positive parental attitudes to participating in research involving newborn screened infants with CF. J Cyst Fibros. 2013;12(3):234-40.

17. Anagnostopoulou P, Yammine S, Schmidt A, Korten I, Kieninger E, Mack I, et al. False normal Lung Clearance Index in infants with cystic fibrosis due to software algorithms. Pediatric

Pulmonology.50(10):970-7. 
18. Stanojevic S, Jensen R, Sundaralingam D, Salazar JG, Yammine S, Singer F, et al. Alternative outcomes for the multiple breath washout in children with CF. J Cyst Fibros. 2015;14(4):490-6.

19. Schibler A, Hall GL, Businger F, Reinmann B, Wildhaber JH, Cernelc M, et al. Measurement of lung volume and ventilation distribution with an ultrasonic flow meter in healthy infants. European Respiratory Journal.20(4):912-8.

20. Gray DM, Willemse L, Alberts A, Simpson S, Sly PD, Hall GL, et al. Lung function in African infants: a pilot study. Pediatr Pulmonol. 2015;50(1):49-54.

21. Gray D, Willemse L, Visagie A, Czovek D, Nduru P, Vanker A, et al. Determinants of early-life lung function in African infants. Thorax. 2017;72(5):445-50.

22. Nguyen TT, Thia LP, Hoo AF, Bush A, Aurora P, Wade A, et al. Evolution of lung function during the first year of life in newborn screened cystic fibrosis infants. Thorax.69(10):910-7.

23. Gaultier C, Fletcher ME, Beardsmore C, England S, Motoyama E. Respiratory function measurements in infants: measurement conditions. Working Group of the European Respiratory Society and the American Thoracic Society. European Respiratory Journal.8(6):1057-66.

24. Hammer Jr, Eber E. Paediatric pulmonary function testing2005.

25. Fuchs SI, Buess C, Lum S, Kozlowska W, Stocks J, Gappa M. Multiple breath washout with a sidestream ultrasonic flow sensor and mass spectrometry: a comparative study. Pediatric Pulmonology.41(12):1218-25.

26. Bayfield KJ, Saunders C, Alton E, Davies JC. P79 Comparison of CF and Non CF LCl results using the Exhalyzer D and InnocorTM devices. Thorax. 2015;70(Suppl 3):A115. 